\title{
Sequencing of a 306 bp Region of the ANXA1 1 Gene Containing the rs 1049550 Polymorphism for Sarcoidosis Susceptibility in Turkish Patients
}

Irfan Degirmenci ${ }^{1}$, Muhsin Ozdemir ${ }^{2}$, Emel Kurt ${ }^{3}$, Tunc Tuncel${ }^{1}$, Faruk Saydam ${ }^{*}$, Oguz Cilingir ${ }^{2}$, Hasan Veysi Gunes ${ }^{1}$ and Sevilhan Artan ${ }^{2}$

${ }^{1}$ Department of Medical Biology, Eskişehir Osmangazi University Faculty of Medicine, Eskişehir, Turkey

${ }^{2}$ Department of Medical Genetics, Eskişehir Osmangazi University Faculty of Medicine, Eskişehir, Turkey

${ }^{3}$ Department of Chest Diseases, Eskişehir Osmangazi University Faculty of Medicine, Eskişehir, Turkey

${ }^{4}$ Department of Medical Biology and Genetics, Recep Tayyip Erdoğan University Faculty of Medicine, Rize, Turkey

\begin{abstract}
Sarcoidosis is a multisystemic immune disorder with unknown etiology. The disease is characterized by the spread of noncaseating epithelioid granulomas. Recent data from Genome Wide Association Studies (GWAS) have identified annexin A11 (ANXA11) as a new sarcoidosis susceptibility gene. These studies further indicated a strong association of a single nucleotide polymorphism (rs1049550) with sarcoidosis. Our aim was to determine whether the rs 1049550 is associated with sarcoidosis in Turkish patients and to scan a 306 bp region of ANXA11 for other variations associated with sarcoidosis. Genomic DNA was isolated from the leukocytes of 53 sarcoidosis patients and 52 controls. A 306 bp region of ANXA11 encompassing rs 1049550 was amplified by PCR, and the amplicons were sequenced using the Sanger method. The sequence data of both patients and controls were analyzed using the BLAST database to identify variations. The allele and genotype frequencies of the groups were analyzed using the chisquare test. The rs 1049550 polymorphism was the only genetic variation observed in the 306 bp region. There was no statistically difference $\left(X^{2}=2.689, P=0.273\right)$ when the frequencies of the CC, CT and TT genotypes in the sarcoidosis group $(58.5 \%, 30.2 \%$ and $11.3 \%$, respectively) were compared with the corresponding genotypes in the control group $(65.4 \%, 17.3 \%$ and $17.3 \%$, respectively). Furthermore, the allele frequencies for the rs 1049550 polymorphism were not significantly different $\left(X^{2}=0.006, P=0.940\right)$ when comparing the sarcoidosis patients $(C=73.6 \%, T=26.4 \%)$ with the controls $(C=74.0 \%, T=26.0 \%)$. Our results suggest that the ANXA11 rs1049550 polymorphism is not a genetic predisposition marker for Turkish patients with sarcoidosis.
\end{abstract}

Keywords: Sarcoidosis; Annexin A11; GWAS; Single nucleotide polymorphism

\section{Introduction}

Sarcoidosis is a multisystemic and polygenic immune disorder [1]. The disease is typically characterized by the presence of noncaseating epithelioid granulomas, particularly in the lungs, skin and eyes [2]. Although the etiology of sarcoidosis remains unknown, recent findings have given researchers important clues about the pathogenesis of the disease. Case control studies have been particularly informative in this regard. According to the sarcoidosis progression hypothesis put forth by ACCESS (A Case Control Etiologic Study of Sarcoidosis), altered immune responses to several environmental, occupational or infectious agents reflect genetic susceptibility and lead to sarcoidosis progression. Furthermore, no singular primary cause of the disease has been identified in the research on sarcoidosis [3]. As the interactions between environmental exposure and genetic susceptibility reflect the etiology of sarcoidosis [3], the genetic basis of the disease will be critical for understanding the molecular pathways underlying disease progression. In this regard, many candidate genes and sarcoidosis-associated polymorphisms have been investigated, and genome scanning studies have uncovered genes strongly associated with the disease along with the corresponding genetic variation [4-6]. In recent years, GenomeWide Association Studies (GWAS), which use millions of genetic markers to analyze whole genomes, have allowed researchers to identify disease-associated loci, variations and mutations [6]. Early findings pertaining to sarcoidosis identified the Major Histocompatibility Complex (MHC) as a susceptibility locus for the disease [6]. Followup studies for the identification of new candidates were subsequently performed. Linkage analyses of MHC loci revealed numerous variants with significant linkage, including the putative susceptibility allele 5 p15.2 and the protective allele 5q11.2 [7,8]. Further analysis of this MHC region revealed a novel susceptibility risk variant in the BTNL2 (butyrophilin-like 2) gene [9], whose association with sarcoidosis was subsequently validated by independent studies in different ethnic groups $[10,11]$. However, whether the BTNL2 variant is independent from HLA alleles remains controversial. Hoffman et al. performed the first sarcoidosis GWAS by genotyping 499 patients and 490 controls using 440,000 SNP markers $[6,12]$. A significant association was identified on chromosome 10, mapping a new sarcoidosis susceptibility gene. This finding was validated by a follow-up GWAS with 1649 patients and 1832 controls. Further analysis of this gene revealed a single nucleotide polymorphism (SNP), rs1049550 (c.688C > T), strongly associated with sarcoidosis [6,12]. This non-synonymous SNP in ANXA11 replaces the arginine at position 230 to a cysteine and is located within a conserved annexin repeat domain in the C-terminal domain [13]. The association of rs 1049550 with sarcoidosis has been validated in studies of independent European populations such as European Africans and Americans [14,15]. Nevertheless, the significance of rs1049550 in terms of protein function and disease association remains unknown. ANXA11 is a member of a vertebrate annexin family of calciumdependent phospholipid-binding proteins and is thought to play a role in the regulation of cytokinesis [16]. However, as mentioned above, the functional significance of the variant in terms of sarcoidosis progression

*Corresponding author: Faruk Saydam, PhD, Department of Medical Biology and Genetics, Recep Tayyip Erdoğan University Faculty of Medicine, 53200, Rize, Turkey, Tel: +90-505-2419232; Fax: +90-4642123000; E-mail: faruk.saydam@gmail.com

Received September 20, 2015; Accepted November 03, 2015; Published November 05, 2015

Citation: Degirmenci I, Ozdemir M, Kurt E, Tuncel T, Saydam F, et al. (2015) Sequencing of a 306 bp Region of the ANXA11 Gene Containing the rs 1049550 Polymorphism for Sarcoidosis Susceptibility in Turkish Patients. Gene Technology 4: 131. doi: 10.4172/2329-6682.1000131

Copyright: (c) 2015 Degirmenci I, et al. This is an open-access article distributed under the terms of the Creative Commons Attribution License, which permits unrestricted use, distribution, and reproduction in any medium, provided the original author and source are credited. 
Citation: Degirmenci I, Ozdemir M, Kurt E, Tuncel T, Saydam F, et al. (2015) Sequencing of a 306 bp Region of the ANXA11 Gene Containing the rs 1049550 Polymorphism for Sarcoidosis Susceptibility in Turkish Patients. Gene Technology 4: 131. doi: 10.4172/2329-6682.1000131

Page 2 of 3

and the underlying mechanism have not been determined. Despite the evidence from genome scanning studies that the ANXA11 rs1049550 SNP is a novel susceptibility variant, it is important to note that studies in different populations may reveal differences in terms of disease association $[14,15]$. Thus, the association of this SNP with sarcoidosis needs to be assessed in different ethnic groups to complement or inform future functional analyses of the molecular mechanisms underlying disease progression.

The aim of the present study was to investigate the association between the ANXA11 rs1049550 SNP and sarcoidosis in a Turkish population and to determine the presence of population-specific SNPs associated with sarcoidosis within the region of interest.

\section{Materials and Methods}

\section{Subjects}

The study subjects were divided into two groups. Subjects with sarcoidosis composed the first group ( $\mathrm{n}=53 ; 40$ women and 13 men), and the mean age was $51.6 \pm 11.3$ years. The second group consisted of healthy controls ( $\mathrm{n}=52 ; 30$ women and 22 men), and the mean age was $49.7 \pm 15.8$ years.

Sarcoidosis was diagnosed based on previously established criteria [17]. The controls were healthy persons with no disease. Informed consent was obtained from all patients and healthy controls in accordance with our protocol, which was approved by the Ethics Committee of the Eskişehir Osmangazi University Faculty of Medicine. The study was conducted in accordance with the ethical principles outlined in the 1975 Declaration of Helsinki. The patients and healthy controls represented a homogeneous Turkish population in terms of ethnicity. Blood samples for the DNA analysis were drawn from the sarcoidosis patients and healthy controls.

\section{Genotyping}

Genomic DNA was isolated from the peripheral leukocytes of the individuals using standard methods [18]. The primer sequences used for the amplification of the $306 \mathrm{bp}$ region of ANXA11 encompassing the rs 1049550 SNP were F- 5'- GCAGAGCATCTCATGACCCCTAC -3 ' and R- 5'- AACGATGCCACCAGCTGGATG -3'. The $50 \mu$ reaction volume contained $45 \mu \mathrm{l}$ master mix and $5 \mathrm{ng}$ genomic DNA. The amplification protocol was as follows: initial denaturation at $95^{\circ} \mathrm{C}$ for 3 minutes; 35 cycles of denaturation at $95^{\circ} \mathrm{C}$ for $30 \mathrm{~s}$, annealing at $59^{\circ} \mathrm{C}$ for $30 \mathrm{~s}$ and extension at $72^{\circ} \mathrm{C}$ for $1 \mathrm{~min}$; and a final extension for 10 minutes. Amplicon quality was controlled by agarose gel electrophoresis (Figure 1). Following the purification protocols, the amplified ANXA11 PCR products were sequenced by DNA cycle sequencing (Applied Biosystems 3130) employing BigDye Terminator chemistry according to the manufacturer's protocols. The genotypes were determined

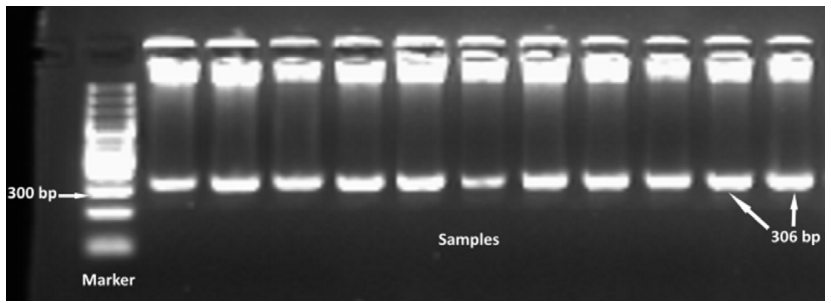

Figure 1: Electrophoresed PCR products on a $2 \%$ gel stained with ethidium bromide were photographed under ultraviolet transillumination. The left lane contains DNA ladder marker (GeneRuler 100 bp DNA Ladder, Thermo Fisher Scientific Inc., MA, USA). Other lanes contain amplified PCR products (306 bp in length) of the samples

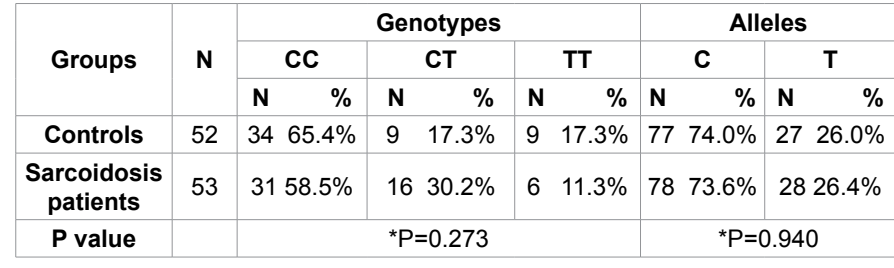

*Pearson's Chi-Square test

Table 1: Genotype and allele frequencies of the ANXA11 rs1049550 polymorphism in sarcoidosis patients and healthy controls

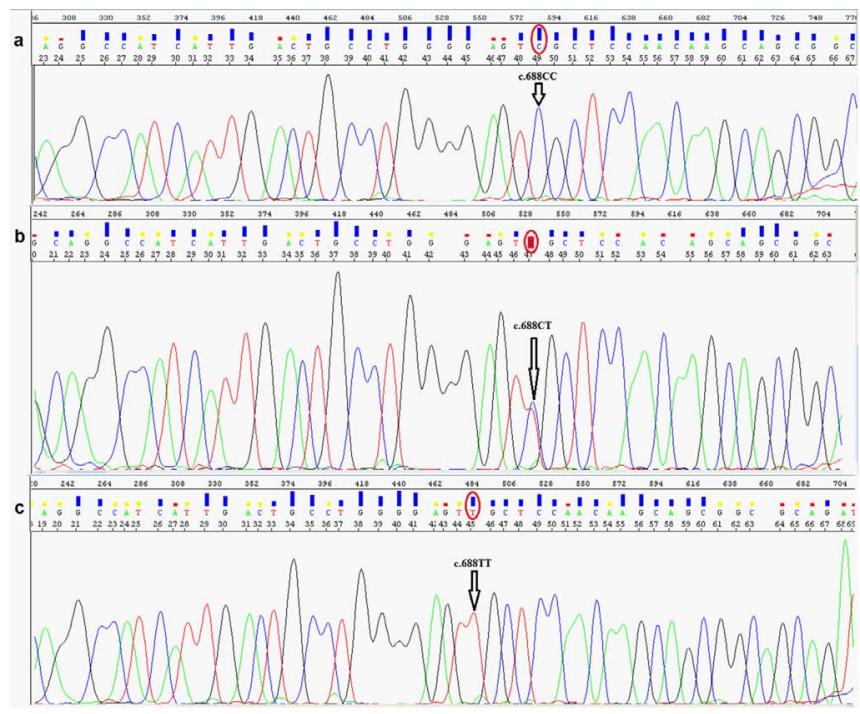

Figure 2: DNA sequence analysis of a $306 \mathrm{bp}$ region of the ANXA11 gene encompassing the rs1049550 polymorphism. a) The homozygous wild-type genotype (CC), b) the heterozygous mutant type genotype (CT) and c) the homozygous mutant type genotype (TT) of the samples for the c.688C $>\mathrm{T}$ polymorphism (rs1049550). Compared with the reference DNA using the BLAST database, there was no variation other than the $c .688 \mathrm{C}>\mathrm{T}$ polymorphism in the sarcoidosis patients.

using Sequence Analysis 5.1 software. For each sample, the genotype was compared to the sequence data from the BLAST database for the identification of any other sequence variations.

\section{Statistical Analysis}

SPSS 15.0 for Windows (SPSS Inc., Chicago, IL, USA) was used for the statistical analysis of the allelic and genotypic distributions. The frequencies of the genotypic and allelic variation in the two groups were compared using the Pearson Chi-Square test.

\section{Results}

The sequence data of both patients and controls were analyzed using the BLAST database to identify variation. The allele frequencies and genotype distributions of the groups were analyzed using the Pearson Chi-Square test (Table 1). The rs1049550 polymorphism was the only genetic variation observed in the $306 \mathrm{bp}$ region sequenced (Figure 2). There was no statistically significant difference $\left(\chi^{2}=2.689\right.$, $\mathrm{P}=0.273$ ) when the frequencies of the $\mathrm{CC}, \mathrm{CT}$ and TT genotypes in the sarcoidosis group (58.5\%, $30.2 \%$ and $11.3 \%$, respectively) were compared with the corresponding genotypes in the control group (65.4\%, 17.3\% and $17.3 \%$, respectively). Furthermore, the allele frequencies for the rs 1049550 polymorphism were not significantly different $\left(\chi^{2}=0.006, \mathrm{P}=0.940\right)$ when comparing the sarcoidosis patients $(\mathrm{C}=73.6 \%, \mathrm{~T}=26.4 \%)$ with the controls $(\mathrm{C}=74.0 \%, \mathrm{~T}=26.0 \%)$. 
Citation: Degirmenci I, Ozdemir M, Kurt E, Tuncel T, Saydam F, et al. (2015) Sequencing of a 306 bp Region of the ANXA11 Gene Containing the rs 1049550 Polymorphism for Sarcoidosis Susceptibility in Turkish Patients. Gene Technology 4: 131. doi: 10.4172/2329-6682.1000131

Page 3 of 3

\section{Discussion}

In the present study, a 306 bp region of the ANXA11 gene encompassing the rs 1049550 SNP was analyzed in Turkish patients with sarcoidosis. This polymorphism was described in the first sarcoidosis GWAS performed by Hoffman et al. [12], which included 499 patients and 490 controls from a German population and utilized 440,000 SNP markers for genotyping. Significant association was detected at chromosome 10q22.3, and this finding was confirmed in an independent cohort study comprising 1649 patients and 1832 controls $[6,12]$. Detailed analysis of ANXA11 revealed a non-synonymous SNP, rs1049550, that converted arginine to cysteine at position 230 [12]. Other risk variants in this region (rs1953600, rs2573346 and rs2784773) were also found to be in strong linkage disequilibrium with rs 1049550 $[6,13,15]$. In the present study, the risk variants rs1953600, rs 2573346 and rs2784773 were not analyzed because they were located outside the sequenced region. The previous findings for rs 1049550 were confirmed by Li et al. [19] in an independent replicative case-control association study of 325 patients and 364 healthy matched controls. Specifically, the frequency of the rs $1049550 \mathrm{C}$ allele was significantly higher in the patient group, and the CC genotype was strongly associated with increased sarcoidosis risk [19]. In addition to this data, no significant differences were found between acute and chronic sarcoidosis for the rs1049550 and rs2573346 alleles, but both groups were found to have allelic and genotypic association for rs1049550 and rs2573346 [19].

ANXA11 and sarcoidosis association has also been investigated in an independent study of African and European Americans. The association of ANXA11 variants including rs1049550 with sarcoidosis and chest radiographic phenotypes was assessed along with HLA variation interactions with ANXA11. A total of 209 SNPs in the promoter, coding and untranslated regions of ANXA11 were genotyped in 1689 patients and 1251 controls. In addition to rs1049550, two new sarcoidosis-associated SNPs, rs61860052 and rs4377299, were identified in African Americans. Specifically, these variants were associated with radiologically classified Scadding stage IV sarcoidosis cases. Additionally, a significant association was found between rs1049550 and rs9268839 near the HLA-DRA locus [15].

ANXA11 protein has critical roles in cellular function, including cell division, calcium signaling, vesicle trafficking, regulation of cytokinesis and apoptosis [3,20,21]. ANXA11 has also been implicated in other autoimmune diseases, such as rheumatoid arthritis, SLE and Sjögren syndrome [21]. The effect of the rs1049550 polymorphism on protein function remains unknown, but it has been proposed to alter the apoptotic characteristics, survival and apoptotic balance of the inflammatory cells [3]. Fatimathas et al. [13] over-expressed GFPtagged ANXA11 in A431 cells and focused on the possible effect of the R230C conversion of rs 1049550 on the cellular distribution and $\mathrm{Ca}^{2+}$ sensitivity of annexin A11. According to their results, the sensitivity of annexin A11 to increases in intracellular calcium is not significantly affected by the sarcoidosis-associated SNP. However, other possibly relevant functions, including those involved in cytokinesis, nuclear envelope breakdown or intracellular signal response, were not evaluated in their study [13].

We found that the ANXA11 rs1049550 polymorphism is not a genetic predisposition marker for Turkish patients with sarcoidosis, and no significant variation was detected in the sequenced region. Due to the heterogeneity among different populations, the effect of this polymorphism on protein function and sarcoidosis progression may remain controversial. The present findings therefore underscore the need to evaluate multiple factors, such as genetic background, environmental exposure and disease subtype, in aggregate. Sarcoidosis shows a very varied incidence among different populations, Turkish people in particular have a very low incidence of the disease [22]. Therefore, the potential limitation of our study is small sample size. A larger sample size would reveal more clearly the present study findings.

\section{References}

1. Muller-Quernheim J, Schürmann M, Hofmann S, Gaede KI, Fischer A, et al. (2009) Genetics of sarcoidosis: a key to understanding its pathogenesis] Pneumologie 63: 166-175.

2. Dempsey OJ, Paterson EW, Kerr KM, Denison AR (2009) Sarcoidosis. BM 339: b3206.

3. Rossman MD, Kreider ME (2007) Lesson learned from ACCESS (A Case Controlled Etiologic Study of Sarcoidosis). Proc Am Thorac Soc 4: 453-456.

4. Hindorff LA, Sethupathy P, Junkins HA, Ramos EM, Mehta JP, et al. (2009) Potential etiologic and functional implications of genome-wide association loci for human diseases and traits. Proc Natl Acad Sci U S A 106: 9362-9367.

5. Manolio TA (2010) Genomewide association studies and assessment of the risk of disease. N Engl J Med 363: 166-176.

6. Spagnolo P, Grunewald J (2013) Recent advances in the genetics of sarcoidosis. J Med Genet 50: 290-297.

7. Gray-McGuire C, Sinha R, lyengar S, Millard C, Rybicki BA, et al. (2006) Genetic characterization and fine mapping of susceptibility loci for sarcoidosis in African Americans on chromosome 5. Hum Genet 120: 420-430.

8. Thompson CL, Rybicki BA, lannuzzi MC, Elston RC, lyengar SK, et al. (2006) Reduction of sample heterogeneity through use of population substructure: an example from a population of African American families with sarcoidosis. American journal of human genetics 79: 606-613.

9. Valentonyte R, Hampe J, Huse K, Rosenstiel P, Albrecht M, et al. (2005) Sarcoidosis is associated with a truncating splice site mutation in BTNL2. Nat Genet 37: 357-364.

10. Spagnolo P, Sato H, Grutters JC, Renzoni EA, Marshall SE, et al. (2007) Analysis of BTNL2 genetic polymorphisms in British and Dutch patients with sarcoidosis. Tissue Antigens 70: 219-227.

11. Rybicki BA, Walewski JL, Maliarik MJ, Kian H, lannuzzi MC; ACCESS Research Group (2005) The BTNL2 gene and sarcoidosis susceptibility in African Americans and Whites. Am J Hum Genet 77: 491-499.

12. Hofmann S, Franke A, Fischer A, Jacobs G, Nothnagel M, et al. (2008) Genome-wide association study identifies ANXA11 as a new susceptibility locus for sarcoidosis. Nat Genet 40: 1103-1106.

13. Fatimathas L, Moss SE (2009) Characterisation of the sarcoidosis-associated variant of annexin A11. Gen Physiol Biophys 28 Spec No Focus: F29-38.

14. Adrianto I, Lin CP, Hale JJ, Levin AM, Datta I, et al. (2012) Genome-wide association study of African and European Americans implicates multiple shared and ethnic specific loci in sarcoidosis susceptibility. PloS one 7: e43907.

15. Levin AM, lannuzzi MC, Montgomery CG, Trudeau S, Datta I, et al. (2013) Association of ANXA11 genetic variation with sarcoidosis in African Americans and European Americans. Genes Immun 14: 13-18.

16. Tomas A, Futter C, Moss SE (2004) Annexin 11 is required for midbody formation and completion of the terminal phase of cytokinesis. J Cell Biol 165: 813-822.

17. Costabel U, Hunninghake GW (1999) ATS/ERS/WASOG statement on sarcoidosis. Sarcoidosis Statement Committee. American Thoracic Society. European Respiratory Society. World Association for Sarcoidosis and Othe Granulomatous Disorders. The European respiratory journal 14:735-737.

18. Gunes HV, Ata N, Degirmenci I, Basaran A, Timuralp B, et al. (2004) Frequency of angiotensin-converting enzyme gene polymorphism in Turkish hypertensive patients. International journal of clinical practice 58: 838-843.

19. Li Y, Pabst S, Kubisch C, Grohe C, Wollnik B, et al. (2010) First independent replication study confirms the strong genetic association of ANXA11 with sarcoidosis. Thorax 65: 939-940.

20. Moss SE, Morgan RO (2004) The annexins. Genome Biol 5: 219.

21. Jorgensen CS, Levantino G, Houen G, Jacobsen S, Halberg P, et al. (2000) Determination of autoantibodies to annexin $\mathrm{XI}$ in systemic autoimmune diseases. Lupus 9: 515-520.

22. Musellim B, Kumbasar OO, Ongen G, Cetinkaya E, Turker H, et al. (2009) Epidemiological features of Turkish patients with sarcoidosis. Resp Med 103: 907-912. 